\title{
The association between patient's and partner's fatigue in couples coping with colorectal cancer: a longitudinal study
}

\author{
M. J. Traa ${ }^{1,2}$ - J. De Vries ${ }^{1,2}$ - J. A. Roukemaa ${ }^{1,3}$ B. L. Den Oudsten ${ }^{1}$
}

Received: 21 January 2016 / Accepted: 17 April 2016 /Published online: 4 May 2016

(C) The Author(s) 2016. This article is published with open access at Springerlink.com

\begin{abstract}
Background Couples coping with colorectal cancer were monitored during the first year after diagnosis to evaluate the following: (i) levels of patients' and partners' fatiguehereby comparing their scores to each other and a normative population, (ii) association between patients' and partners' fatigue, (iii) the course of partners' fatigue, and (iv) biopsychosocial predictors of the partners' fatigue, including the patients' level of fatigue.

Method Couples $(n=171)$ preoperatively completed questions regarding age and sex as well as questionnaires assessing neuroticism and trait anxiety. Questionnaires assessing fatigue, anxiety, and depressive symptoms were measured preoperative (time- 0 ) and 3 (time-1), 6 (time-2), and 12 months (time-3) postoperative. Patients' clinical characteristics were retrieved from the Eindhoven Cancer Registry. Descriptive statistics, correlations, and linear mixed effect models were used.

Results Compared with a normative population, partners' fatigue was similar $(p>.05)$, while patients' fatigue was higher at Time-2 and Time-3 ( $p$ values $<.001$ ). At each time point, correlations between patients' and partners' fatigue were small $(r<.30)$. Partner's course of fatigue was as follows: 18.2 at
\end{abstract}

M. J. Traa

m.j.traa@tilburguniversity.edu

1 CoRPS - Center of Research on Psychology in Somatic Diseases, Department of Medical and Clinical Psychology, Tilburg University, P.O. Box 90153, 5000 LE Tilburg, The Netherlands

2 Department of Medical Psychology, St. Elisabeth Hospital, Tilburg, The Netherlands

3 Department of Surgery, St. Elisabeth Hospital, Tilburg, The Netherlands time-0, 19.0 at time-1, 19.4 at time-2, and 19.2 at time-3 $(p=0.64)$. Scoring higher on neuroticism $(\beta=.12)$ and trait anxiety $(\beta=.23)$, and more depressive symptoms $(\beta=.30)$ significantly contributed to higher partners' fatigue.

Conclusion Trait anxiety, neuroticism, and depressive symptoms predicted higher levels of partners' fatigue, while demographic factors, patients' fatigue, and clinical factors did not. Health professionals are advised to be alert for partners with a vulnerable personality and depressive symptoms. If needed, they can for instance refer to a psychologist for treatment.

Keywords Colorectal $\cdot$ Fatigue $\cdot$ Partners $\cdot$ Interdependency . Caregiver

\section{Introduction}

Fatigue is known as one of the most disturbing and longlasting consequences of cancer treatment. [1-3] The National Comprehensive Cancer Network (NCCN) defined cancer-related fatigue as " . ... distressing persistent, subjective sense of physical, emotional and/or cognitive tiredness related to cancer or cancer treatment that is not proportional to recent activity and interferes with usual functioning." [4] This definition shows that cancer-related fatigue is a subjective, complex, and multidimensional construct. A recent review showed that there are several factors that can influence cancer-related fatigue including genetic risk factors (single nucleotide polymorphisms (SNPs) in inflammation-related genes), psychosocial factors (pre-treatment fatigue, depressive symptoms, sleep disturbance, dysfunctional coping and appraisal processes, loneliness, early life stress), and biobehavioral factors (physical inactivity, elevated body mass index) [5]. The percentage of patients that reports to be fatigued varies across studies, from $15 \%$ [6] to $99 \%$ [7], which is partly due to the 
methodological differences between studies. [8] Cancerrelated fatigue poses challenges on several levels: (i) for the individual, such as being able to adequately perform daily activities [7, 9] and/or an impaired quality of life [10]; (ii) for employers due to decreased work ability and increased sick leave $[11,12]$; and (iii) for the health care system as fatigued patients show an increased health care consumption [13]. Therefore, there are numerous studies dedicated to identifying the level and predictors of cancer-related fatigue [14-16] and evaluating interventions addressing this fatigue using pharmacologic agents [17], exercise [18, 19], or psychosocial counseling [20].

However, a diagnosis and treatment of cancer not only affects the patient but also the partner. [21] Partners also have to incorporate ongoing cancer-related experiences into their lives, as the patients' functioning may influence the partners functioning and vice versa. [21] Moreover, partners are often faced with the care of the patient. A wide variety of caregiving activities exist, which can vary from providing instrumental (e.g., assistance with self-care) to emotional care (e.g., providing social support) [22]. The term caregiver burden is used to describe the burden associated with caring for a loved one. It entails the physical, psychological, social, and/or financial reactions that can be experienced when giving care. [22] In this regard, physical problems, such as pain and sleep problems, are prevalent among partners taking care of the patient [23]. In addition, higher levels of distress have been found in partners of patients with cancer, when comparing them with a normative population. [24-27] Some studies even reported that the distress experienced by the partners is greater than the distress reported by the patients themselves. [28, 29] Furthermore, partners may have to reduce their working capacity in order to provide care, which can result in (additional) financial problems. [30, 31] Finally, partners may experience social challenges, as there is less time and energy available for social interactions. [23] This abovementioned studies show that the biopsychosocial consequences of the patients' illness for the partners have been evaluated to some extent.

However, to our knowledge, limited studies have been conducted evaluating partners' fatigue and the association between patients' and partners' experienced fatigue. This is remarkable considering that fatigue is the most prominent consequence for patients and may, due to the shared experience and bi-directional influence of patients and partners, potentially play a major role for the partners as well. Moderate to severe levels of fatigue were reported in caregivers of patients with cancer [32] as well as a significant positive correlation in the couples levels of fatigue in both patient with breast and prostate cancer [33]. However, the 6-month follow-up study of Peters et al. (2015) found no significant association between patients and caregivers levels of fatigue. [34] However, they did report that fatigue in both patients and caregivers was associated with higher caregiver burden [34].
In addition, Jensen and Given (1993) [35] reported no significant correlation between the severity of fatigue experienced by the caregiver and his/her age, employment status, the number of hours of daily caregiving or its duration, while a significant correlation was found between fatigue of the caregiver and the impact of care on the daily schedule.

However, evaluating partners may be especially interesting as they are most close to the patient. In addition, they often face their own health issues as they are often roughly the same age as the patients. Therefore, they may be especially at risk for developing problems, such as fatigue. Therefore, it is interesting to evaluate the association between patients and partners fatigue and the level of the partners' fatigue over a longer period of time. In addition, biopsychosocial predictors of the partners' fatigue have not yet been identified. The current study aimed to evaluate the following: (i) association between the patients and partners fatigue, (ii) the course of partners' fatigue during the first year after diagnosis, and (iii) biopsychosocial predictors of the partners' fatigue, including the patients' level of fatigue, across time. In order to answer these research questions, a not yet evaluated population was evaluated, i.e., the partners of patients coping with colorectal cancer treated with curative intent.

\section{Methods}

\section{Participants}

Data were drawn from a larger study examining the (sexual) consequences of colorectal cancer for patients and their partners (NCT01234246). For this study, patients and partners were invited to participate. They were recruited from six Dutch hospitals: St. Elisabeth Hospital (Tilburg), TweeSteden Hospital (Tilburg and Waalwijk), Catharina Hospital (Eindhoven), Jeroen Bosch Hospital ('s Hertogenbosch), Amphia Hospital (Breda), and Maxima Medical Centre (Eindhoven and Veldhoven). Patients were excluded if one or more of the following criteria was applicable: (i) elderly age ( $>75$ years), (ii) non-curatively treated metastases at baseline, (iii) poor expression of the Dutch language, (iv) dementia, and/or (v) a history of psychiatric illness. Partners with (i) insufficient knowledge of the Dutch language and (ii) dementia or a history of psychiatric illness were excluded. During a preoperative visit, eligible patients and partners were asked, by their treating physician, if they gave permission to be approached by a member of the research team. Partners were able to participate even if patients declined participation and vice versa, in order to prevent selection bias. Subsequently, a member of the research team contacted potential participants by phone to explain the design and purpose of the study. If patients and/or partners agreed to participate, they were asked to complete a set of questionnaires at home 
before surgery (time-0) and three (time-1), six (time-2), and 12 months (time-3) postoperative. However, at the time of the study, the Dutch guidelines recommended that all patients with rectal cancer, except those with a clinical T1 stage without positive lymph nodes, receive neoadjuvant treatments (i.e., radio(chemo)therapy; www.oncoline.nl). Therefore, a subset of patients and partners completed the first set of questionnaires prior to surgery, but potentially during or after the time patients received neoadjuvant therapy. Patients and partners returned the surveys in sealed postage-paid envelopes. Patients and partners who did not return the questionnaires within 2 weeks received reminders (phone call(s) and/ or a reminder letter). The study was approved by the institutional review board. All participants gave written informed consent. As this specific study evaluated the correlation between patients and partners' levels of fatigue and patientrelated influences on the partners' fatigue, only participating couples were included in the current sample.

\section{Measures}

The patient's clinical information was retrieved from the Eindhoven Cancer Registry (ECR). The ECR routinely collects data on tumor characteristics and treatment. Patients and partners also completed questions regarding their age, sex, and length of the relationship with their partner. The following psychosocial constructs were examined:

For both patients and partners, fatigue was evaluated with the Fatigue Assessment Scale (FAS) [36]. The FAS is a generic questionnaire assessing perceived fatigue and exhaustion with ten items. The response scale is a five-point scale ranging from 1 (never) to 5 (always) and total scores can range from 10 to 50. Total scores can be divided into two groups: not fatigued (a score of 10 to 21) and fatigued (a score of 22 to 50) [36]. The psychometric properties are good. [36]

Two aspects of the partners' personality were assessed. Neuroticism was assessed with the neuroticism facet of the Neuroticism-Extraversion-Openness-Five Factor Inventory (NEO-FFI) [37]. This factor assesses six aspects belonging to neuroticism (i.e., anxiety, hostility, depression, self-consciousness, impulsiveness, vulnerability to stress). Trait anxiety was evaluated with the Dutch short form trait scale of the Spielberger State-Trait Anxiety Inventory (STAI) [38]. The trait anxiety scale describes how persons generally feel and conceives anxiety as a personality disposition [38].

Partners' psychological function was assessed with two constructs. Depressive symptoms were evaluated with the 16-item version of the Center for Epidemiological StudiesDepression Scale (CES-D) [14]. State anxiety was assessed with the short form (6-items) of the STAI state anxiety scale. [39] State anxiety is a momentary emotional condition characterized by subjective feelings of apprehension and tension and heightened autonomic nervous system activity and may thus vary in intensity and fluctuate over time. [39] Sociodemographic factors and personality characteristics were assessed only at time- 0 , while all other questionnaires were completed at each time point (time-0-time-3). The psychometric properties of all questionnaires were satisfactory.

\section{Statistical analyses}

An independent $t$ test and chi-square tests were used to examine potential differences in age, sex, and type of tumor. Pairedsamples $t$ tests were performed to evaluate the level of fatigue between patients and partners. Next, the percentage of fatigued patients and partners at each time point was determined, using the cut-off scores. Levels of fatigue for patients and partners were compared with previously reported normative scores. [36] In addition, bivariate correlations between (i) the levels of fatigue at the different time points for both patients and partners separately and (ii) patients' fatigue and partners' fatigue at each time point were assessed with the Pearson product-moment correlation coefficient. Correlations were grouped into small $(r=<.30)$, moderate $(r=.30-.49)$, or high $(r>.49)$ [40].

Linear mixed-effects models with an unstructured error covariance pattern model were used to examine the course of fatigue and predictors for fatigue. Time was analyzed as a categorical predictor with four levels (i.e., time-0, time-1, time-2, and time-3). The fixed-effects parameters of the models were estimated with maximum likelihood. Sociodemographic variables (i.e., age, sex), patient-related variables (i.e., type of surgery, radiotherapy (yes/no), chemotherapy (yes/no), chemoradiation (yes/no), and the patients' level of fatigue), and personality characteristics (i.e., trait anxiety and neuroticism) were analyzed as time-invariant predictors as they were only assessed at baseline. Psychological variables (i.e., anxiety and depressive symptoms) were measured at each time point and analyzed as time-varying predictors. [41] A forward selection procedure was chosen. In order to determine the final model, separate sets of predictors were evaluated first. The following sets were formed: age and sex (block 1), personality characteristics (block 2), psychological variables (block 3), and clinical characteristics (block 4). These sets were formed based on content. To minimize datadriven choices and to identify the parsimonious model, a $p<.10$ was used during the selection procedure. Next, the selected variables were analyzed in one final model. Furthermore, for this final model, significant effects for the time-varying predictors were split into two effects: betweensubjects effects (e.g., the degree to which patients' fatigue is related to their average level on a predictor) and withinsubjects effects (e.g., the degree to which variation in patients' fatigue over time is associated with a change in their levels on a predictor) [41]. In order to correctly interpret all model parameters, all time-varying variables have been grand-mean 
centered. Analyses were performed in IBM SPSS 22.0, using a significance level of $p<.05$ (with the exception of the selection procedure). Means and standard deviations are reported as mean $\pm \mathrm{SD}$.

\section{Results}

In total, 743 eligible patients agreed to be contacted by a member of the research group, who informed them about the study. Fewer women $(n=280,39 \%)$ than men $(n=445$, $62 \%)$ were approached (for 18 patients $(2 \%)$ information regarding their sex was missing). Of the approached patients, $354(48 \%)$ agreed to participate, $232(66 \%)$ men and 122 (34 \%) women. Various reasons for declining or terminating participation were reported. The most reported reasons for non-participation were (1) the intimate nature of the questionnaires, (2) the length of the questionnaires, and (3) the amount of experienced stress at that time. More men (52\%) than women (43\%) agreed to participate $(p=.033)$. The average age of participants $(62.5 \pm 8.5)$ and non-participants $(63.7 \pm 9.9)$ did not differ $(p=0.84)$. Of the 354 patients, $318(90 \%)$ had a partner of which $229(65 \%)$ participated. In addition, four partners participated even though the patient did not. However, due to the dyadic nature of this study, they were not included. An overview of couples that participated at each time point is presented in Fig. 1. Not all couples completed all assessments. To be included in the analyses couples had to complete at least two assessments. Based on this criterion, $171(75 \%)$ couples were included in the analyses. An overview of the sociodemographic, clinical, and psychological characteristics of the participating couples is presented in Table 1.

\section{Levels of fatigue and correlations between time points and patients and partners}

At time-1 and time-2, partners had significantly lower levels of fatigue than patients (Table 2, $p=.001$ and $p=.004$, respectively). At these time points, approximately one third of the partners scored above the cut-off score for fatigue, as did nearly half of the patients (Table 3 ). Compared with a normative population, the partners' levels of fatigue were comparable at each time point ( $p$ values $>.05$ ). However, patients reported higher levels of fatigue compared with a normative population at time- 1 and time-2 $(p$ values $<.001)$.

For partners, there were high correlations between the levels of fatigue across the several time points ( $r$ values between $r=.53$ and $r=.66$ ), with the exception of the correlation between time- 0 and time- 3 , which was moderate $(r=.36)$. For patients, the correlations between the levels of fatigue were moderate ( $r$ values between $r=.31$ and $r=.50$ ), except for the correlation between time- 0 and time- 1 , which was high $(r=.50)$. Correlations between the levels of fatigue between patients and partners at each time point were low and only significant at time-2 $(r=.20, p=.02)$.

\section{Course and biopsychosocial predictors of the partners' fatigue}

The selection procedure showed that the partners' trait anxiety ( $p<.0001$, block 2$)$, neuroticism $(p=.002$, block 2$)$, state anxiety $(p=.020$, block 3$)$, and depressive symptoms $(p<.0001$, block 3$)$ predicted the course and levels of the partners' fatigue and were, therefore, included in the final model (Table 3, part I). None of the partner's sociodemographic factors (block 1) or the patient's clinical characteristics (block 4 ) predicted the course and levels of the partners' fatigue ( $p$ values $>.05$ ).

In the final model, the estimated marginal means showed that the partner's fatigue at time- 0 was 18.2 , which increased 0.8 points to 19.0 at time- 1 , then increased a little further to 19.4 at time-2, and finally decreased slightly to 19.2 at time- 3 $(p=0.64$, part I, Table 3$)$. Only the partners' fatigue scores at time-2 $(p=.016)$ and time-3 $(p=.030)$ were significantly higher than the partners' fatigue scores at time- 0 . In this mod$\mathrm{el}$, having neuroticism $(\beta=.12, p=.039)$, having trait anxiety $(\beta=.23, p=.015)$, and having depressive symptoms $(\beta=.30$, $p<.0001)$ significantly contributed to a higher partners' fatigue.

The between- and within-subjects analysis for the timevarying predictor depressive symptoms (part II, Table 3) showed that partners who on average had higher levels of depressive symptoms had on average higher levels of fatigue (between-subjects effect, $\beta=.37, p<.0001$ ). Moreover, partners who showed a change in depressive symptoms on a time point also showed a change in fatigue scores (within-subjects effect, $\beta=.26, p<.0001$ ).

\section{Discussion}

From diagnosis until 1 year postoperative, the partners' levels of fatigue were comparable to levels reported in a normative population. Patients reported more fatigue at 3 and 6 months postoperative compared with partners and the normative population. From a clinical perspective, this is not surprising since patients have to cope with cancer-related fatigue. Furthermore, across time correlations between partners' fatigue scores were high, but correlations between the fatigue scores of patients and partners were not. Moreover, the mixed model analyses showed that the patients' levels of fatigue were not indicative of the partners' levels of fatigue. The abovementioned results are not in line with what was expected based on the often reported interdependency between patients and partners. [21] Higher levels of fatigue for the partners 
Fig. 1 Flow-chart of couples that participated at each time point
In total, 229 couples participated

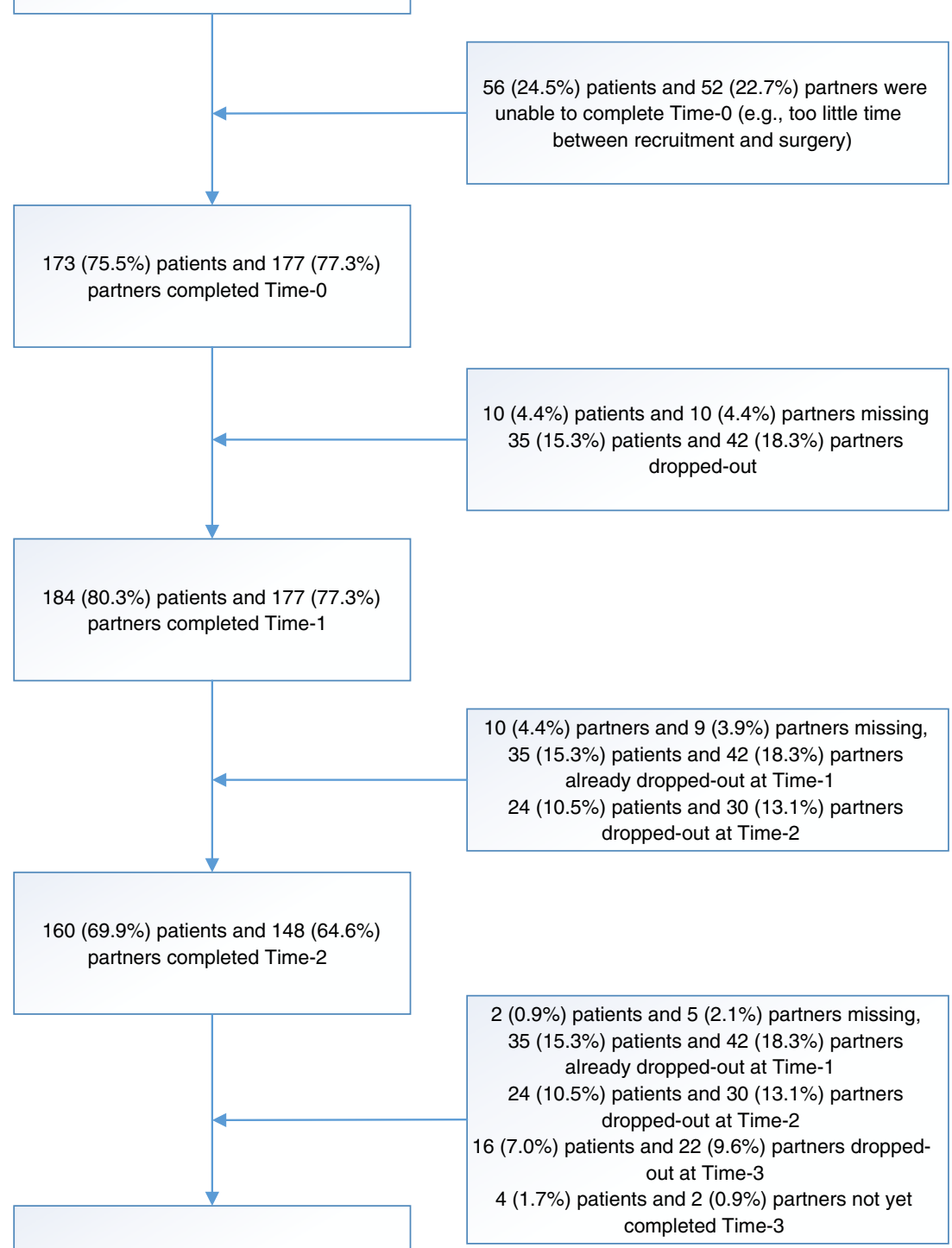

$148(64.6 \%)$ patients and $129(56.3 \%)$ completed Time-3

were expected as partners are faced with the multifaceted care for the patients. Perhaps, partners are reluctant to acknowledge the burden of the patients' disease on their own functioning. Partners may have reported to be less fatigued than they actually were. Especially, since the study did not explicitly focus on fatigue as part of the caregiver burden. Perhaps other factors may play a more prominent role in determining the partners' fatigue, such as daily hassles and the previously reported impact of care on the daily schedule. [35] Two recent studies also reported non-significant correlations between patients and partners' fatigue [34, 42].
The personality characteristics trait anxiety and neuroticism were identified as significant predictors for the partners' fatigue. To our knowledge, this is the first study indicating the role of personality characteristics in partners' functioning. For patients, the influence of trait anxiety [43] and neuroticism [44] on their quality of life has been established. Perhaps, certain personality characteristics, such as trait anxiety and neuroticism, may make people more vulnerable for adverse psychological outcomes, regardless of their status (i.e., patient or partner). The same relationship may apply for depressive symptoms. Depressive symptoms (both between- and within- 
Table 1 Sociodemographic, clinical, and psychological characteristics

\begin{tabular}{|c|c|c|}
\hline & $\begin{array}{l}\text { Partners }(n=171) \\
\text { Mean } \pm \text { SD }\end{array}$ & $\begin{array}{l}\text { Patients }(n=171) \\
\text { Mean } \pm \text { SD }\end{array}$ \\
\hline Age at time of survey & $60.9 \pm 9.3$ & $59.7 \pm 16.1$ \\
\hline Duration of relationship (year) & $35.4 \pm 17.0$ & $34.9 \pm 16.7$ \\
\hline Neuroticism & $29.3 \pm 6.9$ & $27.2 \pm 7.0$ \\
\hline Trait anxiety & $17.3 \pm 4.6$ & $16.6 \pm 4.8$ \\
\hline \multicolumn{3}{|l|}{ State anxiety } \\
\hline Time- 0 & $11.6 \pm 3.3$ & $11.2 \pm 3.4$ \\
\hline Time-1 & $11.1 \pm 3.2$ & $10.2 \pm 3.1$ \\
\hline Time-2 & $11.5 \pm 3.3$ & $10.0 \pm 2.7$ \\
\hline Time-3 & $10.5 \pm 2.8$ & $9.9 \pm 3.2$ \\
\hline \multicolumn{3}{|l|}{ Depressive symptoms } \\
\hline Time-0 & $6.8 \pm 6.5$ & $6.0 \pm 6.7$ \\
\hline Time-1 & $5.8 \pm 6.3$ & $5.7 \pm 5.7$ \\
\hline Time-2 & $5.7 \pm 6.0$ & $5.7 \pm 6.2$ \\
\hline \multirow[t]{2}{*}{ Time-3 } & $5.3 \pm 5.9$ & $4.7 \pm 5.6$ \\
\hline & $n(\%)$ & $n(\%)$ \\
\hline Male sex & $53(31 \%)$ & $160(70 \%)$ \\
\hline \multicolumn{3}{|l|}{ Type of cancer } \\
\hline Colon & - & $72(42 \%)$ \\
\hline Rectum & - & $99(58 \%)$ \\
\hline \multicolumn{3}{|l|}{ Surgery } \\
\hline Low anterior resection & - & $61(36 \%)$ \\
\hline Abdominoperineal resection & - & $32(19 \%)$ \\
\hline Hemicolectomy/ileocecal resection & - & $26(15 \%)$ \\
\hline Sigmoid resection & - & $26(15 \%)$ \\
\hline Other & - & $19(11 \%)$ \\
\hline Unknown type of surgery & - & $7(4$ \%) \\
\hline Radiotherapy, yes & - & $45(26 \%)$ \\
\hline Missing & - & $5(3 \%)$ \\
\hline Chemoradiation, yes & - & $58(34 \%)$ \\
\hline Missing & - & $5(3 \%)$ \\
\hline Chemotherapy, yes & - & $48(28 \%)$ \\
\hline Missing & - & $5(3 \%)$ \\
\hline Stoma, yes & - & $93(55 \%)$ \\
\hline Missing & - & $5(3 \%)$ \\
\hline
\end{tabular}

For eight patients, their clinical characteristics were not available. Neuroticism range $=12-60$. Trait anxiety range $=10-40$. State anxiety range $=4-24$. Depressive symptoms range $=0-48$. Higher scores indicate more problems subjects) were a significant predictor of higher levels of partners' fatigue. For patients, the negative influence of depressive symptoms on fatigue has been reported before $[45,46]$. This relationship was not yet evaluated for partners. However, the depressive symptoms of partners have been associated with their experienced caregiver burden [47]. In addition, there is a well-known overlap of somatic symptoms of depression and symptoms of fatigue, which may also explain this relationship. The patient's treatment-related characteristics did not influence the partners' levels of fatigue. Even though only information on whether or not a patient underwent a certain treatment modality (i.e., surgery, chemotherapy and/or radiotherapy) was available, these variables provided a rough indication of treatment burden. However, as all eligible patients with colon or rectal cancer treated with curative intent were included in this study, a wide variety of treatment schedules occurred. However, the type of treatment patients were still receiving during follow-up (time-1, time-2, and time-3) may have influenced the levels of experienced fatigue. Being unable to clearly incorporate, the heterogeneity in clinical profiles may have prohibited the possibility to adequately determine the precise influence of the several profiles on fatigue. 
Table 2 Fatigue scores for patients and partners

Fatigue scores partners $(\mathrm{M} \pm \mathrm{SD}) \quad$ Fatigue scores patients $(\mathrm{M} \pm \mathrm{SD}) \quad p$ value Percentage fatigued partners Percentage fatigued patients

\begin{tabular}{llllll}
\hline Time-0 & $18.7 \pm 5.2$ & $19.2 \pm 5.56$ & .423 & $19 \%$ & $23 \%$ \\
Time-1 & $20.0 \pm 5.2$ & $22.3 \pm 6.7$ & .001 & $29 \%$ & $46 \%$ \\
Time-2 & $19.7 \pm 5.3$ & $21.7 \pm 7.0$ & .004 & $27 \%$ & $43 \%$ \\
Time-3 & $19.1 \pm 5.5$ & $20.6 \pm 6.5$ & .056 & $22 \%$ & $32 \%$ \\
\hline
\end{tabular}

Statistically significant results $(p<.05)$ are shown in italic

Higher scores indicate more fatigue

Time-0 preoperative measurement, Time-1 3 months follow-up, Time-2 6 months follow-up, Time-3 12 months follow-up, FAS Fatigue Assessment Scale (range 10-50)

Finally, age and gender were no significant predictors for the experienced levels of fatigue. Thus, in this study, the partners' fatigue was not determined by sociodemographic or the patient's clinical characteristics, but only by personality and psychological factors.

There are some additional critical remarks that need to be acknowledged. This data were collected as part of a larger study evaluating the (sexual) consequences of colorectal cancer. The sexual nature of this study made it difficult to recruit participants, resulting in a $45 \%$ response rate. Moreover, a substantial number of drop-outs and/or missing data should be noted. Other remarks concerning the generalizability of the study are that the included sample predominantly consisted of male, heterosexual, Caucasian participants and that elderly patients ( $>75$ years) were not recruited. Furthermore, the preoperative assessment should not be mistaken for a baseline measurement, as the time between diagnosis and surgical treatment can already be psychologically and physically challenging for patients and partners.
Regardless of the abovementioned limitations, this study provided insight in the level of the partners' fatigue over a longer period of time, relatedness between patients and partners' levels of fatigue, and predictors of partners' fatigue. Especially the finding that personality characteristics (i.e., high trait anxiety and neuroticism) and depressive symptoms are indicative of higher levels of partners' fatigue is important. In clinical practice, health professionals are advised to focus not solely on the patient but also to evaluate the functioning of the partners. Especially, partners with a high trait anxiety and neuroticism and depressive symptoms may thus be at risk for fatigue. If psychological care for the partner is needed, health professionals can for instance refer them to a psychologist for adequate and timely treatment. Moreover, optimizing the partners functioning may not only benefit the partner, but the patient as well. Suboptimal adaptation of the partner to the patient's illness may negatively influence the partners' engagement in the patient's care [48]. Furthermore, low partner support can lead to less treatment adherence or fewer favorable lifestyle changes made by the patient [49].
Table 3 Estimates of fixed effects for partners' fatigue

\begin{tabular}{|c|c|c|c|c|c|c|}
\hline & \multirow[t]{2}{*}{ Predictor $^{\mathrm{a}}$} & \multirow[b]{2}{*}{$B$} & \multirow[b]{2}{*}{ SE } & \multirow[b]{2}{*}{ Sig. } & \multicolumn{2}{|l|}{$95 \% \mathrm{CI}$} \\
\hline & & & & & Lower bound & Upper bound \\
\hline \multirow[t]{9}{*}{ Part I } & Time $^{b}$ & & & 0.64 & & \\
\hline & Time-0 (intercept) & 18.19 & .40 & .000 & 17.40 & 19.00 \\
\hline & Time-1-time-0 & .77 & .48 & .113 & -.18 & 1.72 \\
\hline & Time-2-time-0 & 1.18 & .49 & .016 & .23 & 2.14 \\
\hline & Time-3-time-0 & .99 & .46 & .030 & .10 & 1.89 \\
\hline & Neuroticism & .13 & .06 & .039 & .01 & .24 \\
\hline & Trait anxiety & .23 & .09 & .015 & .05 & .41 \\
\hline & State anxiety & .12 & .08 & .134 & -.04 & .29 \\
\hline & Depressive symptoms & .30 & .05 & $<.0001$ & .20 & .39 \\
\hline \multirow[t]{2}{*}{ Part II } & Depressive symptoms: between & .37 & .08 & $<.0001$ & .22 & .52 \\
\hline & Depressive symptoms: within & .26 & .06 & $<.0001$ & .15 & .37 \\
\hline
\end{tabular}

Statistically significant results $(p<.05)$ are shown in italic

Time-0 preoperative measurement, Time-1 3 months follow-up, Time-2 6 months follow-up, Time-3 12 months follow-up

${ }^{a}$ All predictors (except time) are grand-mean centered

${ }^{\mathrm{b}}$ Analysis of variance (ANOVA) type III test 
In this regard, future studies are encouraged to focus not only more specifically on the experienced caregiver burden and the consequences of this burden on the partners (e.g., their biopsychosocial functioning, work ability/sick leave, and health care consumption) but also to evaluate the influence of the partners' biopsychosocial functioning on patient outcomes (e.g., the patients' biopsychosocial functioning, health/illness related-behaviors, and health care consumption).

\section{Conclusion}

Personality characteristics (i.e., high trait anxiety and neuroticism) and depressive symptoms were indicative of higher levels of partners' fatigue. Therefore, health professionals are advised to be alert for partners with a vulnerable personality and depressive symptoms as these partners may be prone to develop problems. If needed, health professionals can refer partners to a psychologist for adequate and timely treatment. Providing care to the partners in need may not only lead to better psychosocial functioning of the partner but may also have favorable outcomes for the patient, via increased partners' engagement in the patient's health.

\section{Compliance with ethical standards}

Conflict of interest The research was funded by the Dutch Cancer Society (UVT 2009-4495). There is no further conflict of interest. The authors have full control of all primary data and allow the journal to review the data if requested.

Open Access This article is distributed under the terms of the Creative Commons Attribution-NonCommercial 4.0 International License (http:// creativecommons.org/licenses/by-nc/4.0/), which permits any noncommercial use, distribution, and reproduction in any medium, provided you give appropriate credit to the original author(s) and the source, provide a link to the Creative Commons license, and indicate if changes were made.

\section{References}

1. Cheng KK, Lee DT (2011) Effects of pain, fatigue, insomnia, and mood disturbance on functional status and quality of life of elderly patients with cancer. Crit Rev Oncol Hematol 78:127-137

2. Ahlberg K, Ekman T, Gaston-Johansson F, et al. (2003) Assessment and management of cancer-related fatigue in adults. Lancet 362: $640-650$

3. Hofman M, Ryan JL, Figueroa-Moseley CD, et al. (2007) Cancerrelated fatigue: the scale of the problem. Oncologist 12(Suppl 1):4-10

4. Mock V, Atkinson A, Barsevick A, et al. (2000) NCCN Practice Guidelines for Cancer-Related Fatigue. Oncology (Williston Park) 14:151-161

5. Bower JE (2014) Cancer-related fatigue - mechanisms, risk factors, and treatments. Nat Rev Clin Oncol 11:597-609
6. Andrykowski MA, Donovan KA, Laronga C, et al. (2010) Prevalence, predictors, and characteristics of off-treatment fatigue in breast cancer survivors. Cancer 116:5740-5748

7. Curt GA, Breitbart W, Cella D, et al. (2000) Impact of cancerrelated fatigue on the lives of patients: new findings from the Fatigue Coalition. Oncologist 5:353-360

8. Husson O, Mols F, van de Poll-Franse L, et al. Variation in fatigue among 6011 (long-term) cancer survivors and a normative population: a study from the population-based PROFILES registry. Support Care Cancer, 2015

9. Mallinson T, Cella D, Cashy J, et al. (2006) Giving meaning to measure: linking self-reported fatigue and function to performance of everyday activities. J Pain Symptom Manag 31:229-241

10. Campos MP, Hassan BJ, Riechelmann R, et al. (2011) Cancerrelated fatigue: a practical review. Ann Oncol 22:1273-1279

11. Duijts SF, van Egmond MP, Spelten E, et al. (2014) Physical and psychosocial problems in cancer survivors beyond return to work: a systematic review. Psychooncology 23:481-492

12. Silver JK, Baima J, Newman R, et al. (2013) Cancer rehabilitation may improve function in survivors and decrease the economic burden of cancer to individuals and society. Work 46:455-472

13. Mewes JC, Steuten LM, Ijzerman MJ, et al. (2012) Effectiveness of multidimensional cancer survivor rehabilitation and costeffectiveness of cancer rehabilitation in general: a systematic review. Oncologist 17:1581-1593

14. Husson O, Mols F, van de Poll-Franse LV, et al. The course of fatigue and its correlates in colorectal cancer survivors: a prospective cohort study of the PROFILES registry. Support Care Cancer, 2015

15. Mitchell SA (2010) Cancer-related fatigue: state of the science. PM R 2:364-383

16. Paiva CE, Paiva BS (2013) Prevalence, predictors, and prognostic impact of fatigue among Brazilian outpatients with advanced cancers. Support Care Cancer 21:1053-1060

17. Minton O, Richardson A, Sharpe M, et al. Drug therapy for the management of cancer-related fatigue. Cochrane Database Syst Rev:CD006704, 2010

18. Cramp F, Daniel J. Exercise for the management of cancer-related fatigue in adults. Cochrane Database Syst Rev:CD006145, 2008

19. Fong DY, Ho JW, Hui BP, et al. (2012) Physical activity for cancer survivors: meta-analysis of randomised controlled trials. BMJ 344:e70

20. Goedendorp MM, Gielissen MF, Verhagen CA, et al. Psychosocial interventions for reducing fatigue during cancer treatment in adults. Cochrane Database Syst Rev:CD006953, 2009

21. Kayser K, Watson LE, Andrade JT (2007) Cancer as a "Wedisease": examining the process of coping from a relational perspective. Fam Syst \& Health 25:404 418

22. Nijboer C, Tempelaar R, Sanderman R, et al. (1998) Cancer and caregiving: the impact on the caregiver's health. Psychooncology 7 : $3-13$

23. Stenberg U, Ruland CM, Miaskowski C (2010) Review of the literature on the effects of caring for a patient with cancer. Psychooncology 19:1013-1025

24. Dumont S, Turgeon J, Allard P, et al. (2006) Caring for a loved one with advanced cancer: determinants of psychological distress in family caregivers. J Palliat Med 9:912-921

25. Hinnen C, Ranchor AV, Sanderman R, et al. (2008) Course of distress in breast cancer patients, their partners, and matched control couples. Ann Behav Med 36:141-148

26. Zenger M, Lehmann-Laue A, Stolzenburg JU, et al. (2010) The relationship of quality of life and distress in prostate cancer patients compared to the general population. Psychosoc Med 30:Doc02

27. Haun MW, Sklenarova H, Villalobos M, et al. (2014) Depression, anxiety and disease-related distress in couples affected by advanced lung cancer. Lung Cancer 86:274-280 
28. Hagedoorn M, Sanderman R, Bolks HN, et al. (2008) Distress in couples coping with cancer: a meta-analysis and critical review of role and gender effects. Psychol Bull 134:1-30

29. Janda M, Steginga S, Dunn J, et al. (2008) Unmet supportive care needs and interest in services among patients with a brain tumour and their carers. Patient Educ Couns 71:251-258

30. Emanuel EJ, Fairclough DL, Slutsman J, et al. (2000) Understanding economic and other burdens of terminal illness: the experience of patients and their caregivers. Ann Intern Med 132:451-459

31. Girgis A, Lambert S, Johnson C, et al. (2013) Physical, psychosocial, relationship, and economic burden of caring for people with cancer: a review. J Oncol Pract 9:197-202

32. Aranda SK, Hayman-White K (2001) Home caregivers of the person with advanced cancer: an Australian perspective. Cancer Nurs 24:300-307

33. Carlson LE, Ottenbreit N, St Pierre M, et al. (2001) Partner understanding of the breast and prostate cancer experience. Cancer Nurs 24:231-239

34. Peters ME, Goedendorp MM, Verhagen SA, et al. (2015) A prospective analysis on fatigue and experienced burden in informal caregivers of cancer patients during cancer treatment in the palliative phase. Acta Oncol 54:500-506

35. Jensen S, Given B (1993) Fatigue affecting family caregivers of cancer patients. Support Care Cancer 1:321-325

36. Michielsen HJ, De Vries J, Van Heck GL (2003) Psychometric qualities of a brief self-rated fatigue measure: the Fatigue Assessment Scale. J Psychosom Res 54:345-352

37. Costa PT, McCrae RR. NEO-PI/FFI manual suplement, Odessa, 1989

38. De Vries J, Van Heck GL (2013) Development of a short version of the Dutch version of the Spielberger STAI trait anxiety scale in women suspected of breast cancer and breast cancer survivors. J Clin Psychol Med Settings 20:215-226
39. Marteau TM, Bekker H (1992) The development of a six-item short-form of the state scale of the Spielberger State-Trait Anxiety Inventory (STAI). Br J Clin Psychol 31(Pt 3):301-306

40. Cohen J (1988) Statistical power analysis for the behavioral scienses. Lawrence Erlbaum, Hillsdalen NJ

41. Hedeker D, Gibbons RD (2006) Longitudinal data analysis. In: Longitudinal data analysis. Wiley, Hoboken NJ

42. Drabe N, Steinert H, Moergeli H, et al. (2016) Perception of treatment burden, psychological distress, and fatigue in thyroid cancer patients and their partners - effects of gender, role, and time since diagnosis. Psychooncology 25:203-209

43. Keyzer-Dekker CM, de Vries J, Mertens MC, et al. (2013) Cancer or no cancer: the influence of trait anxiety and diagnosis on quality of life with breast cancer and benign disease: a prospective, longitudinal study. World J Surg 37:2140-2147

44. Dahl AA (2010) Link between personality and cancer. Future Oncol 6:691-707

45. Andrykowski MA, Schmidt JE, Salsman JM, et al. (2005) Use of a case definition approach to identify cancer-related fatigue in women undergoing adjuvant therapy for breast cancer. J Clin Oncol 23: 6613-6622

46. Goedendorp MM, Gielissen MF, Verhagen CA, et al. (2008) Severe fatigue and related factors in cancer patients before the initiation of treatment. Br J Cancer 99:1408-1414

47. Haj Mohammad N, Walter AW, van Oijen MG, et al. Burden of spousal caregivers of stage II and III esophageal cancer survivors 3 years after treatment with curative intent. Support Care Cancer, 2015

48. Bertoni A, Donato S, Graffigna G, et al. (2015) Engaged patients, engaged partnerships: singles and partners dealing with an acute cardiac event. Psychol Health Med 20:505-517

49. Rankin-Esquer LA, Deeter AK, Taylor C (2000) Coronary artery disease and couples. In: Schmaling KB, Scher T (eds) The psychology of couples and illness: theory, research, and practice. American Psychological Association, Washington DC, pp. 43-70 